\title{
Otitis Media Crónica (OMC) en adultos
}

Dra. Fernanda Pineda Gea Médico general, egresada de la Universidad Católica Redemptoris Mater

UNICA, Managua Audiologa Protésica, egresada del IES-ALBASIT, Albacete, España https://orcid.org/0000-0003-0927-3585

ferpgea@gmail.com

Fecha de recepción: 15 de noviembre, 2019 / Fecha de aceptación: 27 de noviembre, 2019 https://doi.org/10.5377/torreon.v8i22.9026

Palabras clave: Adultos, loratadina en otitis media crónica, otitis media crónica

\section{RESUMEN}

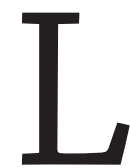

a otitis media crónica es el estado más avanzado en el espectro de las otitis medias, El objetivo de esta investigación fue identificar el tipo de otitis media crónica en adultos nicaragüenses de 25 - 65 años, Para ello se realizó un estudio observacional, descriptivo, de corte transversal en el que se revisaron 312 expedientes clínicos de pacientes diagnosticados con patologías del oído de los cuales 189 tenían diagnóstico de otitis media crónica. Se empleó una técnica de muestreo probabilística sistemática. Se utilizó una ficha de recolección de datos. Las variables sometidas a estudio fueron edad, sexo, tipo de otitis media crónica y tratamiento farmacológico. Para el procesamiento estadístico de estas variables se utilizó el programa informático IBM-SPSS ${ }^{\circledR}$ (Statistical Package for the Social Sciences) versión 25.0 para Windows 7 de 32 bits, se utilizó el paquete de software Microsoft 365 Office ${ }^{\circledR} 2010$ : Microsoft Word ${ }^{\circledR}$ para la depuración de cualquier error de redacción o digitación facilitando de este modo el análisis y reproducción de los resultados y Microsoft Excel ${ }^{\circledR}$ para editar los gráficos y las tablas con el objetivo de hacer más compresible la información obtenida.Se obtuvieron los siguientes resultados; El tipo de otitis media crónica más frecuente fue la otitis media crónica no colestomatosa con proceso infeccioso activo (55\%), independientemente si fuese colestomatosa o no colestomatosa, recibieron como principal tratamiento: loratadina en un $55,4 \%$ ( $n=173$ ), 
Concluyendo que la otitis media crónica no colestomatosa con proceso infeccioso activo es el tipo de otitis media crónica frecuente en nuestro medio.

\section{INTRODUCCIÓN}

La otitis media crónica supone un gasto de salud pública elevado, según lo mencionado por Quintero Noa, Álvarez Lam, Hernández Cordero, Meléndez Quintero (2013) esta entidad clínica presenta un gasto que asciende los 4 billones de dólares estadounidenses por atención médica y cirugía otológica.

La otitis media crónica (OMC) supurada es definida por Quintero Noa y Cols (2013) como un proceso inflamatorio crónico del oído medio sin tendencia a la curación (más de 3 meses), que suele ocasionar lesiones estructurales: secuela cicatrizal definitiva de la membrana timpánica (MT), destrucción y anquilosis de la cadena oscicular, tímpanoesclerosis o colesteatoma.Afecta aproximadamente a 2-3\% de la población mundial según cifras expresadas por Gutiérrez, Godoy, Cárdenas y Argomedo (2016), así mismo, mencionan que no existe predominancia de un sexo.

\section{RESULTADOS}

La otitis media crónica afectó a todos los rangos etarios siendo la el rango etario mayormente afectado en comprendido entre los 51 - 59 años, $17 \%$ ( $n=53)$ seguido del rango etario comprendió entre los 60 - 65 años $13 \%(n=42)$ y en tercer lugar al rango comprendido 25 - 31 años $10 \%$ (n=30). La otitis media crónica afectó al sexo femenino en un 44 \% ( $\mathrm{n}=136)$ y masculino $17 \%(\mathrm{n}=53)$.

La OMC se clasificó tomando en cuenta la presencia o ausencia de colesteatoma relacionado o no a un proceso infeccioso activo o inactivo (Tabla 1), según lo consignado en el expediente, Se observó que de los 189 (100 \%) pacientes diagnosticados con OMC un 167 (88, $3 \%)$ fue clasificado como no colesteatoma. Dentro de la cual, la OMC no colestomatosa activa fue más frecuente (Gráfico 1) en un 55\% ( $\mathrm{n}=104)$ seguida de la OMC no colestomatosa con un $33,3 \%(n=63)$.

Tabla 1. Tipo de otitis media crónica

\begin{tabular}{|c|c|c|c|}
\hline \multirow{6}{*}{ 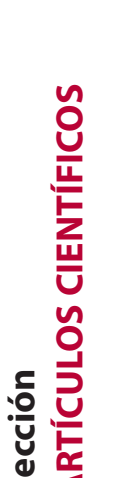 } & Tipo de otitis media crónica & Frecuencia & Porcentaje \\
\hline & OMC* no Colestomatosa - Inactiva & 63 & 33,3 \\
\hline & OMC* no Colestomatosa - Activa & 104 & 55 \\
\hline & OMC Colestomatosa - Activa & 20 & 10,6 \\
\hline & OMC* Colestomatosa - Inactiva & 2 & 1,1 \\
\hline & Total & 189 & 100,0 \\
\hline
\end{tabular}


Nota de la tabla 1: El término activa o inactiva hace referencia a la presencia o no de foco infeccioso en el momento de la consulta. El término colestomatosa hace referencia a la presencia o no de colesteatoma en el oído medio. OMC*: Otitis media crónica. Fuente: Matriz de datos extraídos del expediente clínico.

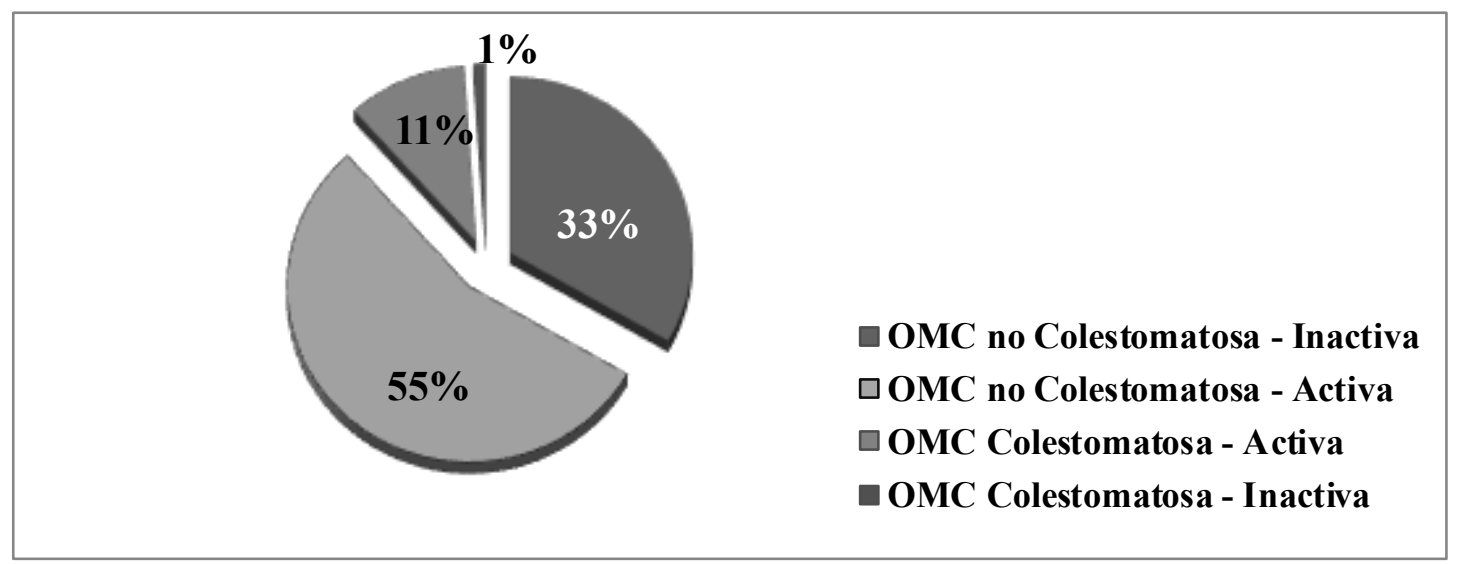

Gráfico 1. Tipo de otitis media crónica. Fuente: Tabla 1.

Los pacientes con OMC asociada o no a otras patologías e independientemente si fuese colestomatosa o no colestomatosa recibieron como principal tratamiento loratadina en un 55,4\% ( $\mathrm{n}=173$ ) (Tabla2). De los pacientes a los que se les prescribió loratadina un 39,7\% (n=122) tenía únicamente OMC.

La loratadina se prescribió según el tipo de otitis (Gráfico 2) en un 57,8\% (n=100) a los pacientes con diagnóstico de OMC no colestomatosa activa y a un 32,4\% ( $\mathrm{n}=56)$ de los pacientes diagnosticados con OMC colestomatosa inactiva.

Tabla 2. Prescripción de loratadina en la otitis media crónica colestomatosa y no colestomatosa en la población estudiada.

Fuente: Matriz de datos extraídos del expediente clínico.

\begin{tabular}{|l|l|l|}
\hline \multirow{2}{*}{ Tipo de otitis media crónica } & \multicolumn{2}{c|}{ Prescripción de loratadina } \\
\cline { 2 - 3 } & \multicolumn{1}{|c|}{ Frecuencia } & Porcentaje \\
\hline OMC $^{*}$ no Colestomatosa - Activa & $\mathbf{1 0 0}$ & $\mathbf{5 7 , 8}$ \\
\hline OMC* no Colestomatosa - Inactiva $^{*}$ & $\mathbf{5 6}$ & $\mathbf{3 2 , 4}$ \\
\hline OMC $^{*}$ Colestomatosa - Activa & 16 & 9,2 \\
\hline OMC* Colestomatosa - Inactiva & 1 & 0,6 \\
\hline TOTAL & 173 & 100,0 \\
\hline
\end{tabular}




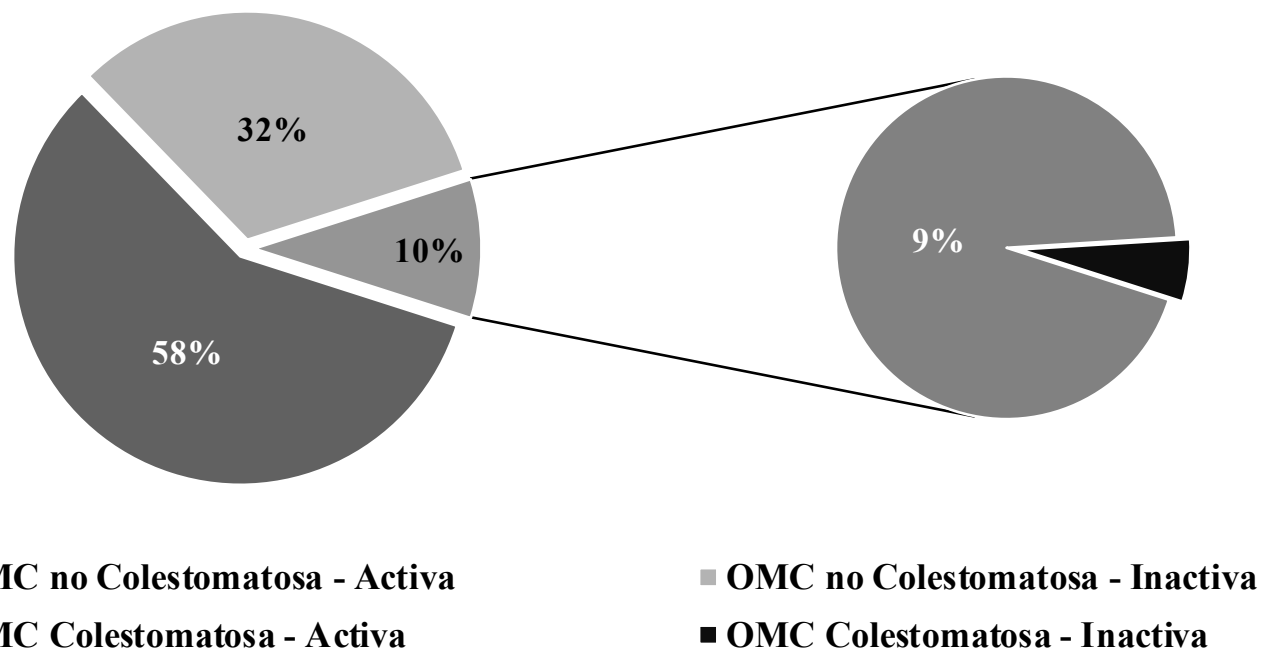

Gráfico 2. Prescripción de loratadina en la otitis media crónica. Fuente: Matriz de datos extraídos del expediente clínico.

\section{DISCUSIÓN}

El rango etario afectado con mayor frecuencia fue el comprendido entre los 51 - 59 años; Se encontró que el sexo femenino se vio mayormente afectado por las otitis medias crónicas, este hallazgo no concuerda con lo reportado por Roig Ocampos Ramallo, JL, Roig Ocampos Forteza, JL, Rossi Vietsky, L, y Mena Caballero, F. (2009) y Chirinos Apaza, M. (2014), quienes expresan que la otitis media crónica es más frecuente en hombres, esta hallazgo es un aporte interesante a la literatura médica ya que confirió a esta investigación una importante relevancia al aportar datos novedosos relacionados al comportamiento de esta patología en nuestro medio.

En este estudio pese a que existen diferentes clasificaciones para la otitis media crónica se consideró la presencia y ausencia de colesteatoma asociada o no con un proceso infeccioso activo. Se reporta que el $90 \%$ de las otitis medias crónicas en estos pacientes era no colestomatosa asociada a un proceso infeccioso activo, concordando con Chinski Alberto y Chiski, Hernan (2009) quienes expresan que esta forma clínica es siete veces más frecuente que la otitis media crónica colestomatosa, la cual es asociada a un bajo nivel socioeconómico y cultural de los pacientes.

El hecho que los procesos infecciosos se hallan asociado a las otitis medias crónicas en nuestro medio evidencia la necesidad de protocolizar el tratamiento de esta patología sobretodo en el abordaje primario, debido a que en la práctica diaria, normalmente los profesionales de atención primaria basan su decisiones terapéuticas en los conocimientos aprendidos durante su formación médica o de indicaciones de otros profesionales lo que podría contribuir al hecho que la discrepancia en el tratamiento primario de esta patología esté relacionada con la tendencia a la cronicidad de la misma. Este hallazgo sugiere la hipótesis que la etiología causal de las otitis 
medias crónicas de origen colestomatosa en nuestro medio se deban a las infecciones otológicas recurrentes y al bajo nivel socioeconómico y cultural de estos pacientes lo que traduce la aparición del colesteatoma, no como una entidad clínica aislada sino como una complicación de una otitis media crónica con evolución tórpida debido a la poca adherencia de los pacientes al tratamiento prescrito; cabe mencionar que muchos autores coinciden que la etiología del colesteatoma es multifactorial.

En lo que respecta a las otitis media crónicas asociada a colesteatoma, se encontró que solo el 10 \% de esta patología se asoció a la presencia de colesteatoma, hecho que refleja el adecuado manejo, diagnóstico y terapéutico del personal médico que labora en el Hospital Escuela Antonio Lenin Fonseca pese al hecho que no exista una normativa estandarizada para el manejo de esta patología en nuestro medio. Llama la atención que la mayoría de los pacientes con otitis media crónica recibieron loratadina, independientemente si esta se asoció o no a un proceso infeccioso, este hecho no concuerda con lo reportado en la bibliografía médica ni con los lineamientos de las guías internacionales para el abordaje y manejo de esta patología, las cuales no reportan datos claros sobre la utilidad de este fármaco en estos pacientes. La adopción de esta práctica médica por parte del personal médico asistencial sugiere la hipótesis de que el prescriptor sea vulnerable a influencias por parte del usuario o familiar de este que al ver el hecho de que su proceso crónico no requiere tratamiento farmacológico influye ejerciendo presión psicológica sobre el prescriptor, el cual termina en una prescripción por complacencia y no basada en criterios clínicos que la fundamenten. Al respecto se sugiere optar por estrategias que favorezcan el uso racional de los medicamentos como lo es la correcta explicación de la técnica de limpieza del conducto auditivo externo (CAE) y su importancia en la evolución de la enfermedad, así como también en la prevención de patologías auditivas asociadas como la otomicosis producida por el ambiente húmedo en el conducto auditivo externo. Estas estrategias contribuirán a la disminución de los costos en salud que genera la prescripción innecesaria de medicamentos y favorecerá el grado de conocimiento de los individuos al concientizarlos de la importancia que trae consigo el autocuido.

Con respecto a la prescripción de antihistamínicos como lo es la loratadina, algunos autores desaconsejan su uso clinico, tal es el caso de Griffin GH, Flynn C, Bailey RE, Schultz JK (2011) quienes reportan que el uso de antihistamínicos en pacientes otitis media no supone ningún beneficio o cierto daño al paciente, ya sea prescrito de manera aislada o en combinación con el tratamiento de la otitis media, de hecho realizaron una recomendación en contra de su uso. Este hallazgo abre la puerta a una línea de investigación enfocada a comprobar la utilidad clínica de este fármaco y el efecto de su uso en pacientes con diagnóstico de otitis media crónica basándose en la hipótesis de que los antihistamínicos como es la loratadina al inhibir los receptores de histamina $\mathrm{H} 1$ favorecen a la disminución de la secreción ótico característica 
de esta entidad clínica, sin embargo para ello se requerirá de un estudio epidemiológico de mayor solidez metodológica. La otitis media crónica en adultos es muy frecuente en nuestro medio e infortunadamente no se cuentan con datos disponibles, exactos y confiables sobre la prevalencia real de esta patología tanto en nuestro medio como a nivel internacional, confiriendo el hecho que los datos aportados en esta investigación servirán como precedentes para futuras investigaciones, lo que permitirá en un futuro estimar no solo la prevalencia sino la incidencia de los caos de otitis media crónica en nuestro.

\section{CONCLUSIONES}

Se concluye que la otitis media crónica no colestomatosa asociada a un proceso infeccioso activo es una patología frecuente en nuestro medio que afecta mayormente a mujeres entre los entre los 51 - 59 años.

Se necesitan más estudios con mayor solidez metodológica como lo es estudio de caso control o ensayo clínico para esclarecer los beneficios o desventajas del uso de loratadina en pacientes con otitis media crónica independientemente se asocie o no colesteatoma con o sin proceso infeccioso activo.

Es necesaria la protocolización del abordaje primario de las patologías auditivas en nuestro medio.

\section{DIVULGACIONES ÉTICAS}

Protección de personas y animales. Los autores declaran que no se realizaron experimentos en humanos o animales para este estudio.

Confidencialidad de los datos: El autor declara que en todo momento se respetó la confidencialidad de la relación médico - paciente registrada en el expediente clínico, ya que en ningún momento durante la recolección de información y divulgación de los resultados se registró el nombre o cualquier información que lo identifique.

Derecho a la privacidad y consentimiento informado: El autor declara que en este estudio la fuente de información fue el expediente clínico y no se tuvo contacto directo con el paciente, por lo que no se solicitó su consentimiento.

\section{REFERENCIAS BIBLIOGRÁFICAS}

Quintero Noa, Julianis Loraine, Álvarez Lam, Ileana, Hernández Cordero, María del Carmen, \& Meléndez Quintero, Loraine Lilia. (2013). Complicaciones de las otitis medias agudas y crónicas en el niño. Revista Cubana de Pediatría, 85(1), 89-105. Recuperado en 12 de noviembre de 2019, de http://scielo.sld.cu/scielo. php? script $=$ sci_arttext $\&$ pid $=$ S003475312013000100009\&lng=es\&tlng=es. 
Gutiérrez, Daniela, Godoy, Ángela, Cárdenas, Juan, Argomedo, Fernando Javier. (2016). Hospital San José Protocolo de referencia y contra referencia en otitis media crónica. Chile. Recuperado en 12 de noviembre de 2019, de https:// docplayer.es/72790919-Hospitalsan-jose-protocolo-de-referencia-ycontrarreferencia-en-otitis-mediacronica.html

Griffin GH, Flynn C, Bailey RE, Schultz JK. (2011). Antihistamínicos y descongestivos para la otitis media exudativa (OME) en niños (Revisión Cochrane traducida). La Biblioteca Cochrane Plus, 2008 Número 4. Oxford: Update Software Ltd. Recuperado en 14 de noviembre de 2019, de http://www. bibliotecacochrane.com. (Traducida de The Cochrane Library, 2008 Issue 3. Chichester, UK: John Wiley \&amp; Sons, $\mathrm{Ltd})$

Chinski Alberto y Chiski, Hernan (2009). Otitis media crónica, VII Manual de otorrinolaringologia pediátrica de la IAPO (Interamericam Association of Pediatric Otorhinolaryngology). Página 230. Recuperado el 14 de noviembre de 2019, de http://www.iapo.org.br/ manuals/VI_manual_es_Alberto\%20 Chinski.pdf
Roig Ocampos Ramallo, JL, Roig Ocampos Forteza, JL, Rossi Vietsky, L, y Mena Caballero, F. (2009). Aspectos clínicos de pacientes con diagnóstico de Otitis Media Crónica. Anales de la Facultad de Ciencias Médicas (Asunción), 42(1), 4550. Recuperado el 14 de noviembre de 2019, de http://scielo.iics.una.py/scielo. php? script $=$ sci_arttext $\&$ pid $=S 1816$ 89492009000100006\&lng=en\&tlng=es.

Chirinos Apaza, M. (2014). Características clínico epidemiológicas de la otitis media crónica colesteatomatosa en el Hospital Regional Honorio Delgado Espinoza Arequipa 2002 - 2012. Arequipa-Perú, Recuperado el 14 de noviembre de 2019, de http://repositorio.unsa.edu. pe/bitstream/handle/UNSA/4282/ MDchapm.pdf? sequence $=1$ \&isAllowed $=$ 